\title{
Building a bioartificial heart: A 3-song saga
}

Philippe Menasché, MD, PhD

From the Department of Cardiovascular Surgery Hôpital Européen Georges Pompidou; INSERM U 970 and Université Paris Descartes, Sorbonne Paris Cité, Paris, France.

Disclosures: Author has nothing to disclose with regard to commercial support.

Received for publication Oct 29, 2016; accepted for publication Oct 31, 2016; available ahead of print Dec 16, 2016.

Address for reprints: Philippe Menasché, MD, PhD, Department of Cardiovascular Surgery, Hôpital Européen Georges Pompidou 20, rue Leblanc, 75015 Paris, France (E-mail: philippe.menasche@aphp.fr).

J Thorac Cardiovasc Surg 2017;153:744-7

$0022-5223 / \$ 36.00$

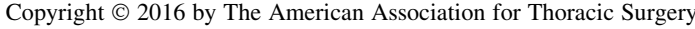

http://dx.doi.org/10.1016/j.jtcvs.2016.10.098

In this issue of the Journal, Lundberg and colleagues ${ }^{1}$ discuss the potential interest, requirements, and challenges of building a bioartificial heart. This clearly written article is seemingly inspired by a recently published article in Science, ${ }^{2}$ in which Park and colleagues describe the development of a robotic, bio-hybrid, light-controlled stingray. How and why could this technologically impressive device be relevant to patients with an end-stage heart failure requiring a cardiac replacement?

\section{ACT I. AQUARIUS (FROM THE MUSICAL HAIR, BY JAMES RADO AND GEROME RAGNI [LYRICS], AND GALT MACDERMOT [MUSIC])}

It seems that the idea of building this bio-hybrid "fish" came to the mind of the senior author of the Science article, ${ }^{2}$ Kevin Kit Parker, when he took his young daughter to the Boston aquarium and was impressed by the similarity of the rhythmic undulations of jellyfishes and the contractions of a human heart. It then took 4 years for Park and his coworkers to develop the product, the design of which can be summarized as follows: A gold skeleton is sandwiched between 2 elastomeric (silicone) layers molded in a shallow cup fitted with flaps. Rat heart cells have then been seeded on the elastomeric sheet that has been coated with fibronectin to promote cell growth according to a radiating pattern reminiscent of the muscles of the fish. Furthermore, these cells have been genetically engineered to express a lightsensitive ion channel that makes them responsive to blue light flashes by a twitch and the subsequent induction of sequential muscular contraction according to a serpentine pattern. In fact, there are 2 light sources directed toward each flap. Changing the frequency of the light flashes allows one to modulate the contraction rate, and making these contractions asymmetric steers the robot toward the right or the left. The gold skeleton acts as a spring that stores elastic energy during the downward contraction of the fins and rebounds once the cells stop contracting. As such, this robotic bio-hybrid that could swim at an average speed of $9 \mathrm{~m}$ per hour and was controlled with enough accuracy to overcome obstacles in its swimming bath illustrates what

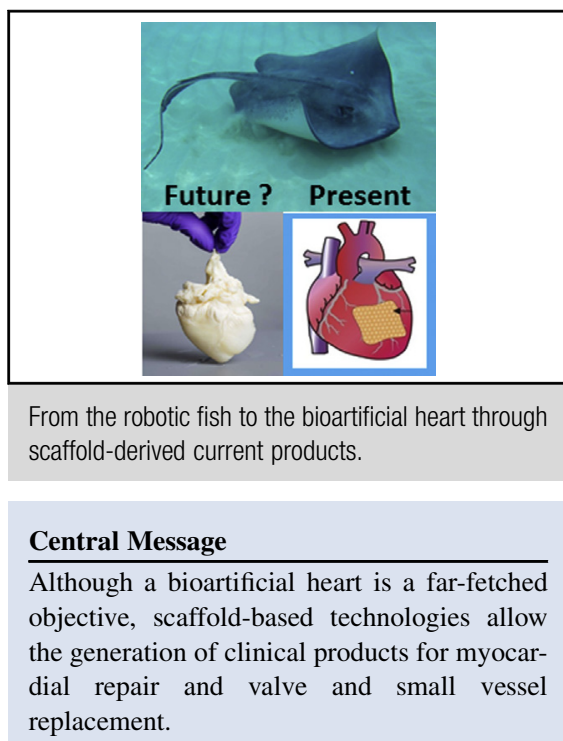

See Article page 748

can be achieved by combining material science, cell biology, and genetics. For Park and colleagues, ${ }^{2}$ this might be the first step toward their ultimate goal, that is, a bio-artificial heart overcoming the issues still associated with heart transplantation or implantation of a total artificial heart in patients with end-stage heart failure. Indeed, as discussed by Lundberg and colleagues ${ }^{1}$ the crude translation of the robotic stingray to the clinics could be envisioned as the patterning of genetically engineered cells onto a scaffold in a way that promotes their coordinated contractions in response to the activation of the encoded gene. Alternatively, the use of naturally contractile cells responsive to electrical stimuli could make them the bio-actuators triggering the scaffold contractions.

\section{ACT II. I HAVE A DREAM (FROM THE SWEDISH POP GROUP ABBA)}

The key features of the robotic fish to be translated into the construction of a bio-artificial heart would be cells acting as both bio-sensors and bio-actuators because of their intrinsic contractile potential, a scaffold providing them with the cues necessary for survival, spatial patterning and contraction, and a cell-responsive activation system. At an organ scale, the most effective approach for generating such a scaffold seems to be perfusion decellularization that yields a cell-free template preserving the tridimensional architecture and the vascular network. ${ }^{3}$ The second stage should be the recellularization of this scaffold by 
the different cell populations that comprise the human heart, that is, primarily cardiomyocytes representing the natural bio-actuators, but also endothelial and smooth muscle cells and fibroblasts. The proof of concept of such an approach has been provided in a seminal article ${ }^{3}$ in which perfusion-decellularized rat hearts subsequently subjected to intramural injections of 50 to $75 \times 10^{6}$ cells (neonatal cardiomyocytes, fibrocytes, endothelial cells, and smooth muscle cells) were able to contract in response to pacing although, importantly, their contractile force represented only $2 \%$ of that of a normal adult rat heart in the rather artificial setting of an in vitro working heart preparation. This clearly illustrates the major technologic challenges of such a bio-hybrid device with regard to its ability to generate enough force to be functionally relevant. Furthermore, although the perfusion-based approach has proven to be successful for generating decellularized scaffolds from human-scale cadaveric organs in the laboratory setting, a clinically relevant, albeit yet unsettled, question is to identify the sterilization method allowing the preservation of the matrix integrity and its capacity to retain structural (ie, pore features), physical (ie, elastic modulus), and chemical (ie, integrin binding sites) signals required for optimal cell functions. ${ }^{4}$ Finally, given the issues associated with the availability of scaffolds from nondiseased human hearts, a xenogeneic source should be considered. The feasibility of such an approach, from both the processing and the regulatory standpoints, is now illustrated by the current clinical evaluation of a decellularized porcine extracellular matrix discussed next. Lundberg and colleagues ${ }^{1}$ also appropriately mention that an alternate theoretically possible approach could be to start from a "plain" scaffold and to mold it as a human heart because of the technologic advances in 3-dimensional printing. However, this optimistic perspective needs to be tempered by the observation that bioink performances and bioprinter resolution do not yet allow to leverage current tridimensional molding to a large-sized multiscale structure with proper spatial patterning and chamber-specific distribution of cells, regulation of microenvironmental matrix-associated physico-chemical cues, and functionally effective vascular tree networks. ${ }^{5}$

Recellularization is equally daunting. Using the patient's own cells is conceptually attractive but likely unrealistic because of the complexity, and consequently the cost of manufacturing patient-tailored organs. Allogeneic cells probably would be required and as appropriately pointed out by Lundberg and colleagues, ${ }^{1}$ induced pluripotent stem cells are attractive candidates because of their straightforward availability, scale-up potential, and ability to be differentiated toward the desired lineages according to the composition of the culture media and the additional use of mechanical or electrical conditioning. Furthermore, the use of major histocompatibility complex-matched cells might theoretically mitigate the expected immune response, whereas bioreactors could allow to repopulate the scaffold with the huge number of cells required to match that of a human heart. However, one should not underestimate that such a process would be labor-intensive, expensive, and still fraught with the risk that a large-scale production may complicate control over a consistent cellular phenotype; more important, it would also be critical not only to replenish the scaffold but also to ensure that the candidate cells orient properly and cross-talk in a way that provides an adequate function at the tissue level. The ability to achieve such a goal remains to be shown. In the study by Lu and colleagues, ${ }^{6}$ decellularized mouse hearts were successfully recellularized with induced pluripotent stem cell-derived multipotent cardiovascular progenitors. The cues provided by both the matrix and the exogenously supplied growth factors were effective in driving the commitment of cells toward the cardiac and endothelial lineages; the resulting constructs demonstrated spontaneous contractions and generated a calcium-responsive contractile force. However, the ability of this bio-artificial heart to function in vivo, even in an heterotopic setting, was not tested. Moving to large-scale organs, Welman and colleagues $^{7}$ have reported that decellularized porcine scaffolds, recellularized by intracoronary infusion of human umbilical cord-derived endothelial cells and intramural injections of murine neonatal cardiac cells, could display myocardial electrical activity after 10 days of bioreactor perfusion, but here again, the functional correlate was not assessed, even ex vivo. Two recent studies have finally looked at human hearts. The article by Garreta and colleagues $^{8}$ claims the "rapid production of human heart grafts," but the methodology actually consisted, after successful decellularization of whole cadaver human hearts, of seeding thick slices of the myocardium with pluripotent stem cell-derived "cardiac-like" cells that were then shown to exhibit a coordinate electromechanical activity. In the study by Guyette and colleagues, ${ }^{9}$ acellular human hearts were seeded with approximately 500 million intramyocardially injected, human-induced, pluripotent stem cell-derived cardiomyocytes in a bioreactor providing both coronary perfusion and mechanical stimulation of the left ventricular wall. Although the electrically paced reseeded scaffolds exhibited contractions, developed pressures were only in the range of $2.4 \pm 0.1 \mathrm{~mm} \mathrm{Hg}$, and less than, or at most, $50 \%$ of the reseeded areas were populated by cardiomyocytes of varying degrees of maturity. Thus, all these studies share in common the lack of current evidence that the successfully recellularized constructs are able to sustain the workload on an in vivo heart. Clearly, much work needs to be done and should specifically include optimization of culture conditions to yield mature cardiomyocytes; development of techniques to achieve both full recellularization of the matrix and the 
valvular scaffold and reendothelialization of the vascular tree allowing an adequate blood supply while avoiding clot formation; better characterization of sterilization and storage methods; and assessment of the in vivo immune response of the matrix to the host microenvironment.

\section{BACK TO THE EARTH (LYRICS BY JASON MRAZ)}

Although construction of a total bio-artificial heart is likely a far-fetched objective, "intermediate" therapeutic products could benefit from advances in the tissueengineering technologies provided their translational development integrates 3 main interconnected considerations: ease and consistency of manufacturing, compliance with regulatory guidelines, and affordable cost. Indeed, some of these products are there, but they only target regeneration of a portion of the heart, that is, a discrete myocardial area, a valvular apparatus, or a vascular conduit. As outlined earlier, the recellularization step with exogenous cells looks like the most complicated part of the process at a wholeorgan scale, but the challenge is not insurmountable when downgraded to a smaller area. Dohmen and colleagues ${ }^{10}$ have reported excellent 10-year results in a series of 11 patients who underwent placement of a cryopreserved pulmonary allograft that had been previously decellularized, coated, and seeded with autologous vascular endothelial cells. However, issues associated with cell procurement, manufacturing, and regulation have made it attractive to skip ex vivo scaffold reseeding with exogenous cells and to rely on endogenous cells to repopulate it. The composition of the acellular scaffold, either naïve or functionalized with bioactive peptides, ${ }^{4}$ then becomes critical to provide the cells with the most appropriate physical and chemical cues required for their adherence, survival, proliferation, and differentiation. This objective is realistic and illustrated by the clinical use of such products for repair of scarred myocardium, valve replacement, and revascularization (Figure 1). Thus, in the context of ischemic heart failure, an acellular decellularized porcine extracellular matrix (VentriGel; Ventrix, San Diego, Calif) is currently undergoing a phase I trial with the premise, based on a robust array of preclinical data, that it could trigger endogenous cardioreparative responses involving angiogenesis, limitation of cardiomyocyte apoptosis, inflammation and fibrosis, and recruitment of host-associated cardiac progenitor cells. ${ }^{11}$ In the valve area, acceptable outcomes have been yielded by the CryoValve SG decellularized (and not in vitro cell-reseeded) pulmonary valves (CryoLife, Kennesaw, $\mathrm{Ga}$ ) in patients undergoing the Ross procedure or right ventricular outflow tract reconstruction. ${ }^{12}$ In line with this kind of approach, the company Xeltis (Zürich, Switzerland) has reported at the last meeting of the European Association for Cardiothoracic Surgery that 3 pediatric patients requiring reconstruction had been enrolled in the "Xplore-I" clinical

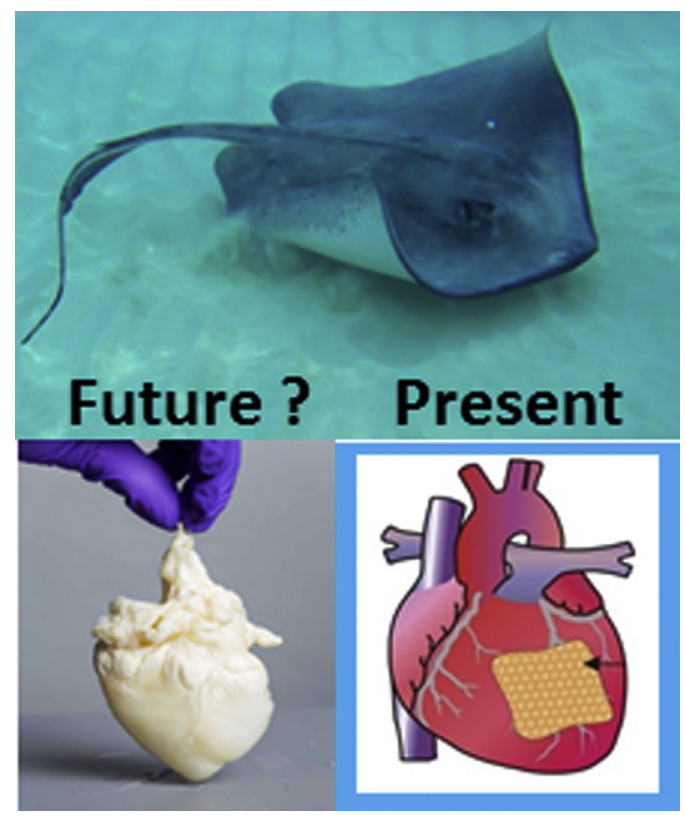

FIGURE 1. From the robotic fish to the bioartificial heart through scaffold-derived current products (a myocardial patch is shown).

study of a bioabsorbable pulmonary heart valve (in this device, the decellularized scaffold has been replaced by a synthetic material based on a proprietary technology, but the concept of fostering endogenous cell sources for generating a living conduit is the same). In the field of vascular reconstruction, another slightly different approach has been adopted that consists of skipping the use of a whole or partial organ scaffold and to start from a cultured cell-derived matrix. Small-caliber vascular conduits have been generated by first seeding banked vascular smooth muscle cells onto a tubular scaffold that degrades as the cells are secreting their own matrix, followed by a decellularization step that lets a natural conduit. Finally, this conduit can be seeded with endothelial cells or functionalized with defined proteins recapitulating the repertoire of integrin receptors to promote attachment of circulating cells. ${ }^{13}$ The Humanity Trial (NCT02644941), sponsored by Humacyte (Morrisville, NC), is currently recruiting patients with end-stage renal failure requiring hemodialysis (target number 350) for assessing the effects of these bioengineered human acellular vessels (Humacyl) as arteriovenous shunts in comparison with expanded polytetrafluoroethylene grafts.

Overall, these approaches are appealing because they rely on a well-defined, straightforward, and cost-effective manufacturing process. Whether, in the long term, decellularized scaffolds can be advantageously replaced by biomimetic polymers remains to be determined. In the meantime it is important to further optimize the procedures for natural extracellular matrix decellularization to both preserve cell-specific cues and eliminate any residual 
immunogenic epitope. ${ }^{14}$ The cautionary words recently raised about the inflammatory response occasionally elicited by the broadly used Cormatrix scaffold ${ }^{15}$ support this requirement for devices providing the most biomimetic niche-like template. Such an achievement would be a relevant step in the ongoing development of a totally bioartificial heart.

\section{References}

1. Lundberg MS, Baldwin JT, Buxton DB. Building a bio-artificial heart: obstacles and opportunities. J Thorac Cardiovasc Surg. 2017;153:748-50.

2. Park SJ, Gazzola M, Park KS, Park S, Di Santo V, Blevins EL, et al. Phototactic guidance of a tissue-engineered soft-robotic ray. Science. 2016;353:158-62.

3. Ott HC, Matthiesen TS, Goh SK, Black LD, Kren SM, Netoff TI, et al. Perfusion-decellularized matrix: using nature's platform to engineer a bioartificial heart. Nat Med. 2008;14:213-21.

4. Rana D, Zreiqat H, Benkirane-Jessel N, Ramakrishna S, Ramalingam M. Development of decellularized scaffolds for stem cell-driven tissue engineering. J Tissue Eng Regen Med. June 29, 2015 [Epub ahead of print].

5. Duan B. State-of-the-art review of 3D bioprinting for cardiovascular tissue engineering. Ann Biomed Eng. April 11, 2016 [Epub ahead of print].

6. Lu TY, Lin B, Kim J, Sullivan M, Tobita K, Salama G, et al. Repopulation of decellularized mouse heart with human induced pluripotent stem cell-derived cardiovascular progenitor cells. Nat Commun. 2013;4:2307.
7. Welman T, Michel S, Segaren N, Shanmugarajah K. Bioengineering for organ transplantation: Progress and challenges. Bioengineered. 2015;6:257-61.

8. Garreta E, de Oñate L, Fernández-Santos ME, Oria R, Tarantino C, Climent AM, et al. Myocardial commitment from human pluripotent stem cells: rapid production of human heart grafts. Biomaterials. 2016;98:64-78.

9. Guyette JP, Charest JM, Mills RW, Jank BJ, Moser PT, Gilpin SE, et al Bioengineering human myocardium on native extracellular matrix. Circ Res. 2016;118:56-72.

10. Dohmen PM, Lembcke A, Holinski S, Pruss A, Konertz W. Ten years of clinical results with a tissue-engineered pulmonary valve. Ann Thorac Surg. 2011;92: $1308-14$.

11. Wassenaar JW, Gaetani R, Garcia JJ, Braden RL, Luo CG, Huang D, et al Evidence for mechanisms underlying the functional benefits of a myocardial matrix hydrogel for post-MI treatment. J Am Coll Cardiol. 2016;67:1074-86.

12. Brown JW, Elkins RC, Clarke DR, Tweddell JS, Huddleston CB, Doty JR, et al Performance of the CryoValve SG human decellularized pulmonary valve in 342 patients relative to the conventional CryoValve at a mean follow-up of four years. J Thorac Cardiovasc Surg. 2010;139:339-48.

13. Dahl SL, Kypson AP, Lawson JH, Blum JL, Strader JT, Li Y, et al Readily available tissue-engineered vascular grafts. Sci Transl Med. 2011;3: $68 \mathrm{ra9}$.

14. Song JJ, Ott HC. Organ engineering based on decellularized matrix scaffolds Trends Mol Med. 2011:17:424-32.

15. Nehzad ZM, Poncelet A, de Kerchove L, Gianello P, Fervaille C, El Khoury G. Small intestinal submucosa extracellular matrix (CorMatrix $\left.{ }^{\circledR}\right)$ in cardiovascular surgery: a systematic review. Interact Cardiovasc Thorac Surg. 2016;322: 839-50. 